\title{
DISTRIBUCIÓN ACTUAL Y POTENCIAL DE LAS CACTÁCEAS FEROCACTUS HISTRIX, MAMMILLARIA BOMBYCINA Y $M$. PEREZDELAROSAE EN EL ESTADO DE AGUASCALIENTES, MÉXICO
}

\author{
Ernestina Meza-Rangel ${ }^{1}$, Felipe Tafoya ${ }^{2}$, Roberto Lindig-Cisneros ${ }^{3}$, José Jesús \\ Sigala-Rodríguez ${ }^{2}$, Eugenio PéRez-Molphe-Balch ${ }^{1,4}$ \\ ${ }^{1}$ Universidad Autónoma de Aguascalientes, Centro de Ciencias Básicas, \\ Departamento de Química, Avenida Universidad 940, Ciudad Universitaria, \\ 20100 Aguascalientes, Aguascalientes, México. \\ ${ }^{2}$ Universidad Autónoma de Aguascalientes, Centro de Ciencias Básicas, \\ Departamento de Biología, Avenida Universidad 940, Ciudad Universitaria, \\ 20100 Aguascalientes, Aguascalientes, México. \\ ${ }^{3}$ Universidad Nacional Autónoma de México, Centro de Investigaciones en \\ Ecosistemas, Antigua Carretera a Pátzcuaro Núm. 8701, Colonia Ex Hacienda de \\ San José de la Huerta, 58190 Morelia, Michoacán, México. \\ 4Autor para la correspondencia: eperezmb@correo.uaa.mx
}

\section{RESUMEN}

Las cactáceas en México son un grupo representativo de las zonas áridas que actualmente presenta problemas de conservación debido a la sobreexplotación y el saqueo de los que han sido objeto en todo el país. En este trabajo se reporta la distribución actual de tres especies de cactáceas en el estado de Aguascalientes actualmente sujetas a protección especial según la legislación ambiental federal mexicana (Ferocactus histrix, Mammillaria bombycina y M. perezdelarosae). Se estimó su distribución potencial mediante la técnica de modelado de nicho ecológico. La mayoría de las poblaciones de $F$. histrix se encontraron en la mitad oeste del estado de Aguascalientes, entre 1750 y 2473 m s.n.m., en cañadas de difícil acceso o en laderas con poca inclinación. Las de M. bombycina fueron encontradas en la parte suroeste del de la entidad, entre 1847 y $2417 \mathrm{~m}$ de altitud, en colonias en paredes de cañadas o a ras del suelo cerca de las cañadas. Por último, las de $M$. perezdelarosae se ubicaron en el suroeste y el centro del estado, en rocas muy expuestas, entre 2024 y $2417 \mathrm{~m}$. El estudio aporta una actualización al conocimiento de la distribución y proporciona nuevos elementos para conocer la condición de conservación de estas especies en Aguascalientes y para buscar estrategias de reintroducción y conservación. 
Palabras clave: biznaga, Cactaceae, conservación, modelado de nicho ecológico.

\begin{abstract}
The Cactaceae is a representative group of the national flora of Mexico that faces conservation problems due to overexploitation and illegal trade at the national and state levels. In this paper the present distribution of three cactus species protected by Federal Law (Ferocactus histrix, Mammillaria bombycina and M. perezdelarosae) occurring in the state of Aguascalientes is updated and their potential distribution estimated using ecological niche modeling techniques. Most populations of $F$. histrix were found in the western half of the state of Aguascalientes at elevations between 1750 and $2473 \mathrm{~m}$, in canyons of difficult access or low hills. The populations of $M$. bombycina were located in southwestern Aguascalientes at elevations between 1847 and $2417 \mathrm{~m}$, in canyon walls and in horizontal terrain close to the cliffs. Finally, populations of $M$. perezdelarosae were located in the southwestern and central regions of the state on exposed bedrock at elevations between 2024 and $2417 \mathrm{~m}$. This study contributes to our knowledge of the distribution and the populations of all three species, which may be used for future reintroduction and conservation efforts of these species in the state of Aguascalientes.
\end{abstract}

Key words: barrel cactus, Cactaceae, conservation, ecological niche modeling.

\title{
INTRODUCCIÓN
}

Los mapas de distribución son esenciales para el estudio de los patrones de distribución de las plantas y son la base para el establecimiento de estrategias de conservación, dado que no es posible proteger una especie sin saber dónde está presente. Los que se usan con mayor frecuencia son los de puntos y superficies sombreadas, pero tienen varias desventajas ya que subestiman la distribución real en regiones que no han sido muestreadas o sobrestiman aquellas que lo han sido de manera deficiente (Anderson et al., 2002). El modelado de nicho ecológico es una técnica que se usa para identificar áreas de distribución potencial con base en variables ambientales, información de presencia de las especies y algoritmos que estiman el nicho climático usando información ambiental y de localidad (Guisan y Zimmermann, 2000; Graham et al., 2004). A pesar de las imprecisiones intrínsecas del modelado (Pearson et al., 2006), tiene una gran diversidad de aplicaciones que van de la 
teoría de conservación del nicho y especiación (Peterson et al., 1999; Wiens, 2004), el cambio climático (Peterson et al., 2002; Ballesteros-Barrera et al., 2007), procesos de invasión de las especies (Peterson, 2003), el muestreo de especies raras (Guisan et al., 2006; Pearson et al., 2007), la agricultura (Sánchez-Cordero y Martínez-Meyer, 2000), la salud humana y animal (Peterson y Kluza, 2003; Peterson et al., 2005) o bien la exclusión y la expansión de nicho ecológico de una especie, asociada con la falta de competencia con otras (liberación competitiva) (Anderson et al., 2002), entre muchas otras (Kozak et al., 2008).

Por la diversidad de su fisiografía, clima y suelos del país, la flora de México es una de las más ricas y variadas del continente. Una de las familias que mejor la representa es la de las cactáceas (Bravo Hollis, 1978), las cuales se encuentran principalmente en zonas áridas y semiáridas, y son utilizadas para elaborar jugos concentrados, postres, jarabes, mermeladas y deshidratados, y como plantas ornamentales.

Actualmente muchas de las actividades del hombre están causando la disminución de las poblaciones de cactáceas, con acciones tales como la destrucción de su hábitat por asentamientos humanos, el saqueo ilegal o la introducción de animales en los lugares donde crecen. Estas plantas son especialmente vulnerables debido a su distribución geográfica restringida, sus ciclos de vida largos y sus tasas bajas de crecimiento (Hernández y Godínez, 1994; Godínez-Álvarez et al., 2003).

Bravo Hollis (1978) presenta una perspectiva general y a nivel nacional del estado de conservación de las cactáceas mexicanas, pero existe la necesidad de realizar estudios de menor escala que puedan ser más informativos y útiles para la toma de decisiones a nivel local. Elegimos el estado de Aguascalientes como un caso particular debido a tres razones: hay información base sobre la distribución de las cactáceas en la entidad, la presencia de una diversidad biológica alta en la entidad (Ávila, 2008) y el pequeño tamaño de la misma (5589 km²; Anónimo, 1995), lo que permite una evaluación rápida pero completa de la distribución de cactáceas con problemas de conservación.

Aguascalientes está situado en el centro de la República Mexicana. El estado posee un clima semiseco principalmente y en él se tienen registrados 12 géneros y 70 especies de cactáceas (Quezada et al., 2000); de ellas, Mammillaria bombycina Quehl y M. perezdelarosae Bravo \& Scheinvar son nativas de Aguascalientes, demandadas como ornamentales, mientras que Ferocactus histrix (DC) G. E. Linds es característica del centro de México, usada en la producción de dulces, agua de biznaga y como planta ornamental. De acuerdo con la NOM-059-SEMARNAT-2010 (Anónimo, 2010), las tres especies se encuentran sujetas a protección especial. Además, a nivel regional la sobreexplotación, aunada a la destrucción de su hábitat, ha 
hecho que la situación de conservación de estas especies se agrave (Anderson, 2001; Del Castillo y Trujillo, 1991).

Para el estado de Aguascalientes existen pocos estudios sobre cactáceas. De la Cerda (1999) menciona que $F$. histrix es escasa, crece en el matorral subtropical en el municipio de Calvillo (en el suroeste de la entidad); además registra a $M$. bombycina para selva baja caducifolia en el mismo municipio y a M. perezdelarosae como una especie sumamente escasa en el matorral subtropical de Calvillo, en el bosque de encino en San José de Gracia (en el noreste) y en el matorral rosetófilo de Jesús María (en la porción central). Quezada et al. (2000) amplían la distribución publicada por De la Cerda (1999) para F. histrix, pues la reportan también de los municipios de Asientos, Cosío, Rincón de Romos, Pabellón, Jesús María, San José de Gracia y Tepezalá; incluyen asimismo dos localidades para M. perezdelarosae en los municipios de San José de Gracia y Calvillo, pero no lo hacen para M. bombycina.

Se actualiza la información sobre la distribución de las tres especies en el estado y se estima su distribucnión potencial utilizando la técnica de modelado de nicho ecológico. El propósito de este estudio es identificar y delimitar aquellas áreas que pudieran tomarse en consideración para una futura introducción de individuos de estas especies obtenidos por medios biotecnológicos.

\section{MATERIALES Y MÉTODOS}

La recolección de información se hizo mediante recorridos quincenales a diferentes localidades del estado de Aguascalientes entre enero de 2009 y febrero de 2010 en busca de las especies estudiadas. Los lugares de muestreo se definieron a partir de los datos obtenidos en la literatura (Fitz-Maurice, 1989; De la Cerda, 1999; Pilbeam, 1999; Quezada et al., 2000; Pilbeam y Bowdery, 2005; Quezada, 2008;) y de la consulta de las bases de datos en linea Cactus and Succulent Field Numbre Finder y de la Unidad de Informática para la Biodiversidad (UNIBIO-UNAM). Se revisaron los herbarios de la Universidad Autónoma de Aguascalientes y del Instituto Nacional de Investigaciones Forestales, Agrícolas y Pecuarias en Pabellón, Aguascalientes. Las exploraciones fueron planeadas para cubrir la mayor parte de las regiones del estado, incluso aquellas en donde no se habián reportado estas especies. El metodo de búsqueda fue a través de transectos de longitud variable que cubrían diferentes tipos de vegetación y formas topográficas. Para cada ejemplar encontrado se registraron las coordenadas precisas usando un receptor de posicionamiento satelital GPS Garmin ETrex (Datum WGS83) y se tomó nota de las caracterñisticas del 
microhábitat inmediato: (a) vegetación, (b) presencia de otros cactus, (c) presencia de plantas nodrizas, y (d) cercanía a rocas.

Para el modelado de nicho ecológico se empleó el software MaxEnt (3.3.2, febrero de 2010, Princeton University \& AT\&T Labs-Research, Princeton, NJ, descarga gratuita en http://www.cs.princeton.edu/ schapire/maxent/), el cual toma como base para predecir la distribución de una especie en un área geográfica en el conjunto de ubicaciones de ocurrencia georreferenciado (todas las coordenadas de las localidades visitadas) y las variables ambientales (Phillips et al., 2004; Phillips et al., 2006; Phillips y Dudík, 2008). Estas últimas fueron elegidas del paquete de 19 variables ambientales de escala mundial llamado Worldclim, generadas por Hijmans et al. (2005) y disponibles en http://www.worldclim.org.

Se realizó un primer modelado para delimitar el área de estudio con bases biológicas y geográficas usando el sistema de información geográfica Manifold (versión 8.0.16, Ultimate Edition, Carson City, NV, marzo 2010) en el que se integraron los puntos recopilados para revisarlos en busca de posibles errores y se elaboraron los mapas de localidades de colecta. Con esta delimitación se recortaron las capas ambientales de Worldclim de manera individual. Se obtuvo así un plano en el que se asignan valores continuos cuantitativos a cada pixel del área de estudio, que fue transformado en uno binario en el que indica la presencia potencial de las especies de interés en Aguascaliente. Esta transformación se hizo mediante el método de Maximización de la suma de sensibilidad-especificidad (Liu et al., 2005). Posteriormente éste se combinó con información del hábitat de las especies obtenida durante las salidas de campo y con la cartografía de vegetación y uso de suelo 1:250 000 (CONABIO, 1999) para identificar los tipos de vegetación en los cuales estaban los puntos de colecta de las tres especies y así corroborar las observaciones de campo. Tal procedimiento tuvo la finalidad de hacer más precisos los mapas de distribución potencial.

\section{RESULTADOS}

Las localidades visitadas en la fase del trabajo de campo en donde se encontraron ejemplares de las especies en estudio fueron 76 para $F$. histrix, 30 para $M$. bombycina y 60 para M. perezdelarosae; muchas de ellas son sitios de los que estas plantas no habían sido reportadas previamente. En la figura 1 se muestran los lugares en los que fueron halladas (algunos puntos están superpuestos por lo que no se aprecian bien en el mapa). Ferocactus histrix se presenta en el norte y este de Aguascalientes, en los municipios de Asientos, Calvillo, El Llano, Jesús María, Rincón de 
Romos, San José de Gracia y Tepezalá; para el El Llano no se había registrado con anterioridad. Mammillaria bombycina se localizó en el suroeste en los municipios de Calvillo y Jesús María, siendo este último también un lugar de donde no se había reportado esta especie. En el caso de M. perezdelarosae, ésta fue encontrada en los municipios de Aguascalientes, Asientos, Calvillo, Jesús María, San Francisco de los Romo y San José de Gracia; destacando los de Aguascalientes, Asientos, Jesús María y Rincón de Romos como áreas en donde no se había registrado.

Se obtuvieron varios modelos con el programa MaxEnt para cada especie con la finalidad de ajustar condiciones, eliminar variables ambientales redundantes y mejorar la capacidad predictiva de los mismos. Las figuras 2, 3 y 4 muestran los mapas finales producidos por el modelado de nicho ecológico para cada una de ellas. Los tipos de vegetación en los cuales se ubicaron los puntos de colecta de las tres especies fueron los siguientes: $46.96 \%$ de los ejemplares de $F$. histrix tuvieron una correspondencia con el matorral subtropical, $24.24 \%$ con el pastizal natural, $21.21 \%$ con el matorral sarcocrasicaule, $4.54 \%$ con el bosque de encino y $3.03 \%$ con áreas de manejo agropecuario. Para M. bombycina $73.33 \%$ de los ejemplares se presentaron en áreas de manejo agropecuario ocupadas con anterioridad por matorral subtropical principalmente, $13.33 \%$ en matorral subtropical y $10 \%$ en bosque de encino. De M. perezdelarosae $46.66 \%$ fueron localizados en bosque de encino, $26.66 \%$ en pastizal, $21.66 \%$ en matorral subtropical y $5 \%$ en áreas de manejo agropecuario (Fig. 5). Cabe mencionar que con frecuencia nuestra interpretación del tipo de vegetación observado durante las salidas de campo fue diferente del mostrado por la carta de la CONABIO (1999). De acuerdo con los modelos construidos en MaxEnt se identificaron las variables que tuvieron más importancia para explicar la distribución de esta especie: para F. histrix fueron el intervalo medio de temperatura semanal, la estacionalidad de la precipitación, la temperatura mínima del periodo más frío del año, la precipitación del cuatrimestre más húmedo y la estacionalidad de la temperatura; sin embargo, fueron las dos primeras las que contribuyeron de manera más importante a la construcción del modelo. Para M. bombycina las variables más significativas fueron la temperatura mínima del periodo más frío del año, la precipitación del cuatrimestre más caliente, el intervalo de temperatura anual y la precipitación del mes más seco. Finalmente, para M. perezdelarosae se identificaron la precipitación del cuatrimestre más caliente, la temperatura mínima del periodo más frío, la estacionalidad de la precipitación y la precipitación del cuatrimestre más frío del año.

Preferencias de hábitat de las especies

Ferocactus histrix crece en altitudes de entre 1750 y 2473 m, en cañadas, cerca de rocas o sobre ellas. Fue notorio que en las localidades que se visitaron en 
Meza-Rangel et al.: Distribución actual y potencial de cactáceas en Aguascalientes

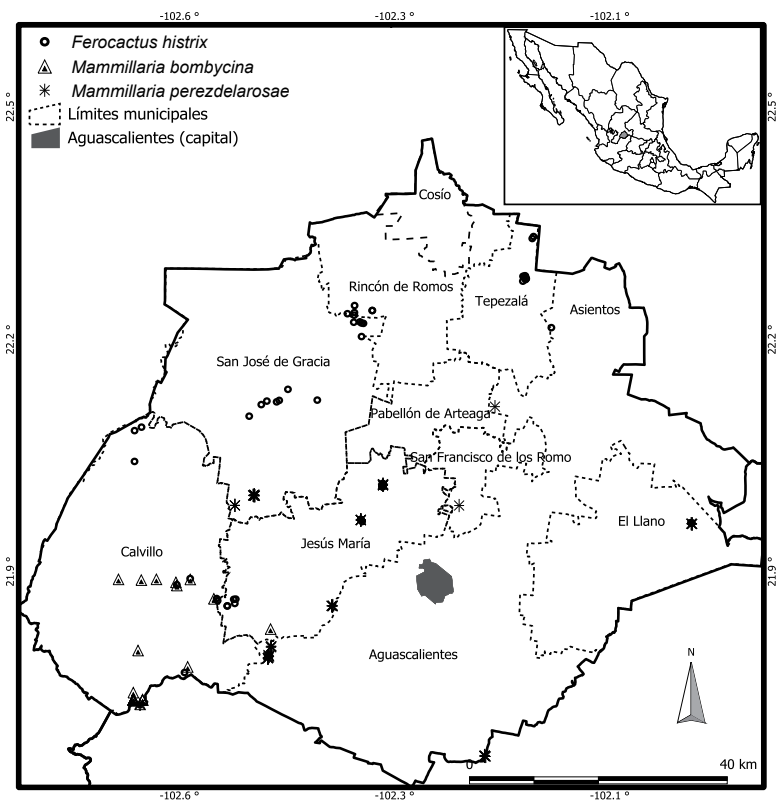

Fig. 1. Distribución de las tres especies en el estado de Aguascalientes, México.

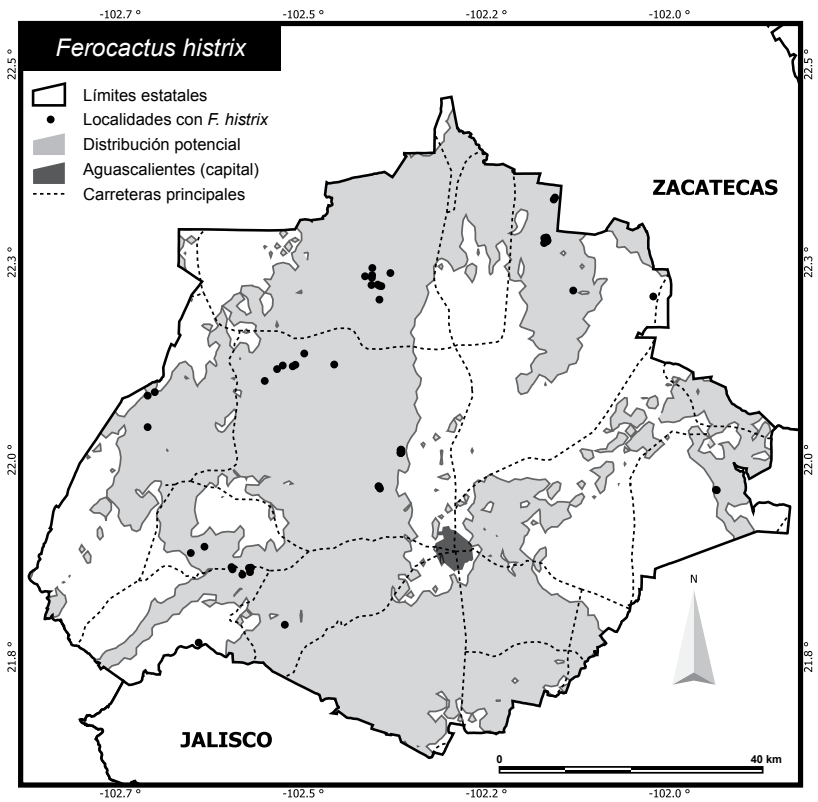

Fig. 2. Mapa de distribución potencial de Ferocactus histrix en el estado de Aguascalientes, México. 


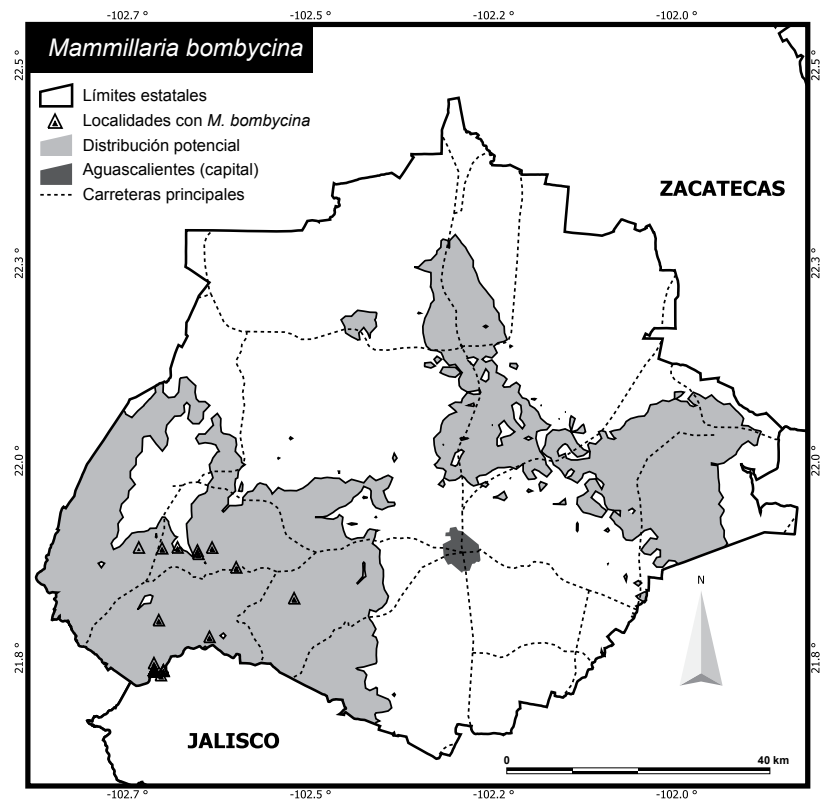

Fig. 3. Mapa de distribución potencial de Mammillaria bombycina en el estado de Aguascalientes, México.

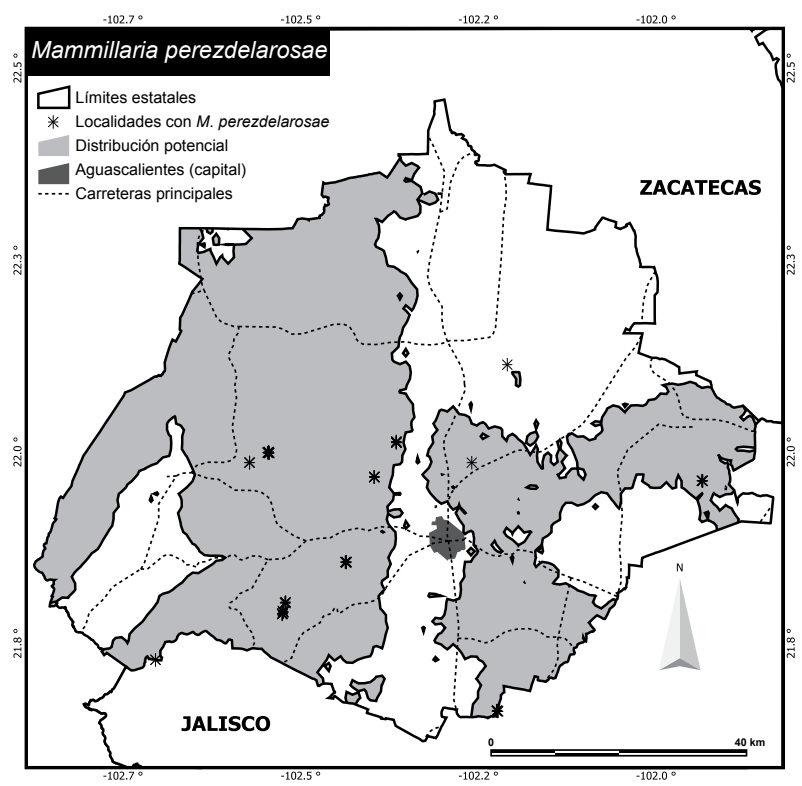

Fig. 4. Mapa de distribución potencial de Mammillaria perezdelarosae en el estado de Aguascalientes, México. 
Meza-Rangel et al.: Distribución actual y potencial de cactáceas en Aguascalientes

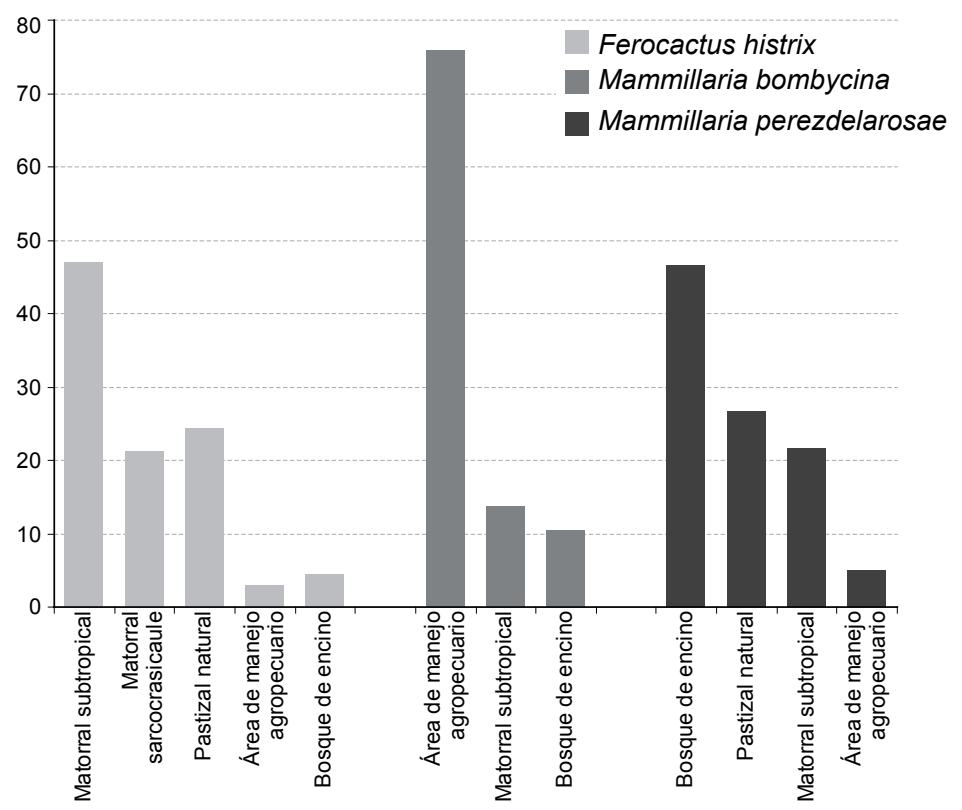

Fig. 5. Distribución en porcentajes de los puntos de colecta en los tipos de vegetación en los que se encontró a Ferocactus histrix, Mammillaria bombycina y Mammillaria perezdelarosae.

el noroeste del estado se observaron individuos en laderas con poca inclinación, mientras que los encontrados en la mitad oeste del mismo estaban generalmente en cañadas de difícil acceso (Fig. 6-A).

Las plantas de $F$. histrix se pueden encontrar en una condición solitaria o formando grupos de dos o tres ejemplares, frecuentemente cerca de plantas arbustivas como Mimosa sp. (uña de gato), Acacia sp. (huizache) y Prosopis sp. (mezquite). En algunas ocasiones este cacto está asociado a Mammillaria bombycina en los sitios en donde sus áreas de distribución coinciden.

Se encontraron varias colonias de Mammillaria bombycina en altitudes de 1847 a $2417 \mathrm{~m}$, algunas en cañadas y otras al nivel del suelo en la base de paredes rocosas o en la unión de rocas (Fig. 6-B). Crece tanto en zonas con suelos húmedos frecuentemente asociada a briofitas (musgos) y Quercus rugosa (encino), como en zonas más secas en asociación con gramíneas y otras cactáceas.

Mammillaria perezdelarosae se encontró en altitudes entre 2024 y $2417 \mathrm{~m}$, creciendo sobre rocas muy expuestas, comúnmente asociada con briofitas (musgos), Selaginella sp. (siempre viva), líquenes, o gramíneas; forma colonias de pocos ejemplares y fue común hallar ejemplares solitarios (Fig. 6-C). 

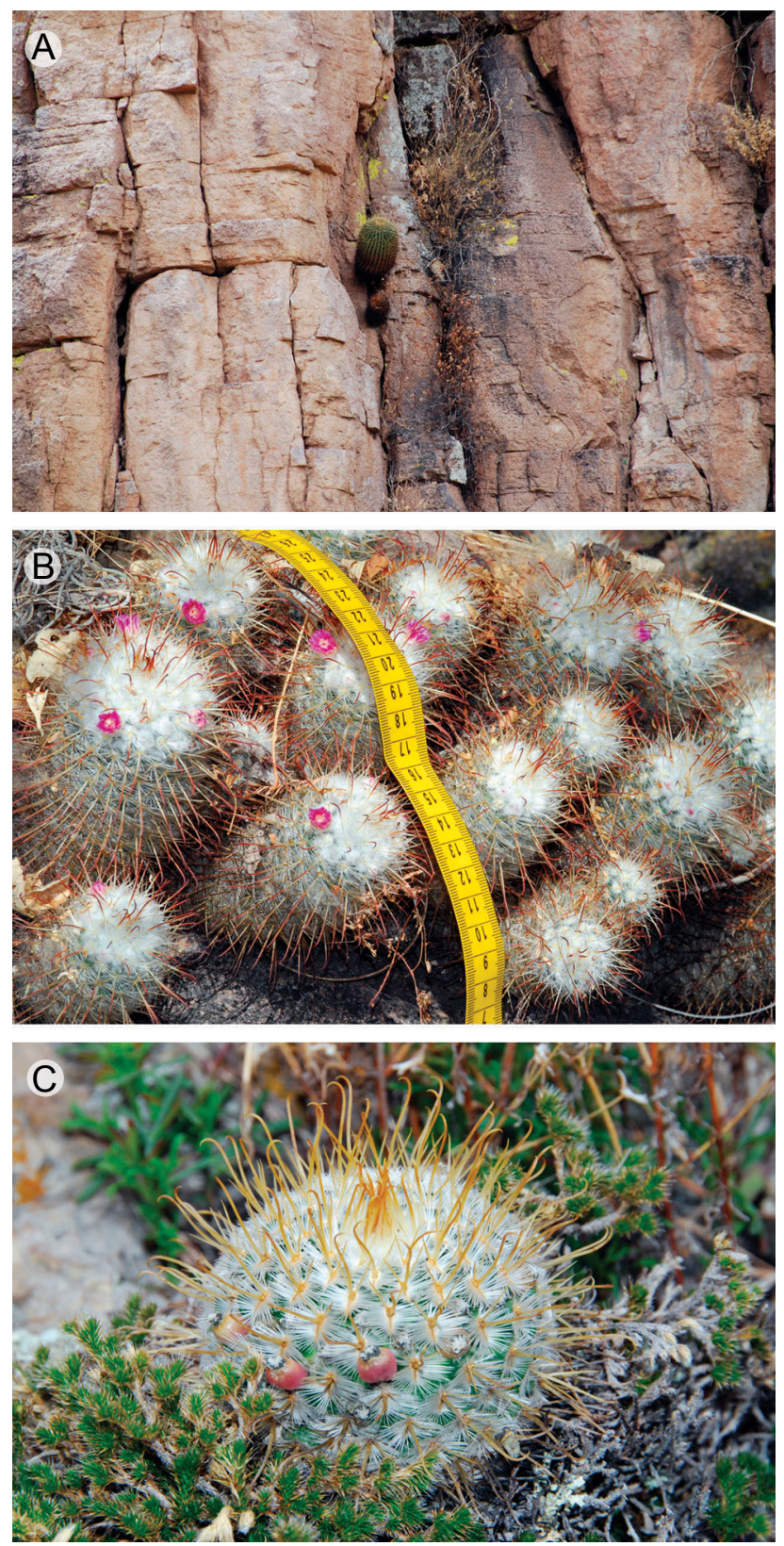

Fig. 6. Imágenes de las especies de estudio en el estado de Aguascalientes; A. Ferocactus histrix en Potrerillos; el ejemplar en la parte central de la imagen tiene un diámetro aproximado de 30 cm.; B. Mammillaria bombycina en Calvillo; C. Mammillaria perezdelarosae en Los Planes; este ejemplar tiene un diámetro aproximado de $7 \mathrm{~cm}$. 


\section{DISCUSIÓN}

A partir de las visitas realizadas al campo, se logró identificar la importancia que tienen las plantas nodrizas en el desarrollo de las cactáceas estudiadas en estado juvenil (Valiente-Banuet et al., 1991). Se observaron ejemplares pequeños de $F$. histrix en varias de las localidades visitadas, especialmente en Cerro de Juan Grande en el municipio de Palo Alto y en la cañada de Potrerillos en el municipio de Rincón de Romos, lo que es indicativo de nuevos reclutamientos en esas poblaciones. También se supo, a través del contacto directo con pobladores locales, que el dulce de biznaga que se comercializa en el estado se trae de estados vecinos, gracias a lo cual ha disminuido la presión de colecta sobre estas poblaciones a nivel local. Sin embargo, la presencia de ganado es común en la región y sigue teniendo un impacto negativo en la mayoría de las zonas visitadas. Por otro lado, ante la escasez de alimento por la sequía presente en algunas áreas del estado, algunos roedores consumen los individuos jóvenes y las semillas de estas plantas.

Los resultados del trabajo de campo permitieron ampliar las distribuciones conocidas de las especies de estudio en comparación con lo reportado por De la Cerda (1999) y Quezada et al. (2000). Además, se reconocen las características de preferencia de hábitat para estas especies, derivadas de lo observado en las salidas de campo y las asociaciones que establecen con sus plantas nodrizas. Por estas razones, este estudio puede tomarse como base para posteriores investigaciones de índole ecológico que contribuyan a confirmar la presencia o ausencia de las especies en las zonas de la distribución potencial.

La estimación de la distribución potencial de las tres especies por medio de un ejercicio de modelación es un avance importante para plantear estrategias de conservación, y tal información puede ser usada en iniciativas de reintroducción en áreas naturales.

\section{CONCLUSIONES}

Se localizó un número importante de poblaciones de las tres especies estudiadas en el estado de Aguascalientes, con lo que se amplió y se actualizó el conocimiento de su distribución en la entidad. Los datos recabados en el estudio de campo se agregan a los disponibles en la literatura y proporcionan un mejor conocimiento de las características del hábitat que ocupan cada una de ellas, lo cual es de importancia fundamental en cualquier estrategia de conservación en sus áreas naturales 
de distribución. A partir de los mapas de distribución potencial de F. histrix, M. bombycina y M. perezdelarosae para el estado obtenidos mediante el modelado de nicho ecológico, se pudieron identificar los sitios con las condiciones ambientales más apropiadas para cada una de estas especies, lo cual puede ser de utilidad para futuras iniciativas de producción y reintroducción de cactáceas.

\section{AGRADECIMIENTOS}

A Margarita De la Cerda Lemus, Octavio Rosales Carrillo, Gabriel González Adame, Gerardo García Regalado, Roberto Rico Martínez, Alberto Rodríguez Ávalos, Silvestre Delgadillo, Guadalupe Mata Pérez, Martha Evelia Pérez Reyes, Isaac Reyes Silva, Daniela Cano, Sofia Sigala Meza y Eric Sigala Meza por su ayuda en campo, en la revisión del manuscrito y por su apoyo personal. A dos revisores anónimos cuyos comentarios incrementaron significativamente la calidad del manuscrito. Al Consejo Nacional de Ciencia y Tecnología por la beca para estudios de doctorado para E.M.R.

\section{LITERATURA CITADA}

Anderson, E. F. 2001. The cactus family. Timber Press. Portland, U.S.A. 776 pp.

Anderson, R. P., A. T. Peterson y M. Gómez-Laverde. 2002. Using niche based GIS modeling to test geographic predictions of competitive exclusion and competitive release in South American pocket mice. Oikos 98: 3-16.

Anónimo. 1995. Estadísticas del medio ambiente. Instituto Nacional de Estadística, Geografía e Informática (INEGI). Aguascalientes, México. 447 pp.

Anónimo. 2010. Norma Oficial Mexicana. NOM-059-SEMARNAT-2010. Protección ambiental. Especies nativas de México de flora y fauna silvestres. Categorías de riesgo. Categorías para su inclusión, exclusión o cambio. Lista de especies en Riesgo. Diario Oficial de la Federación. Secretaría del Medio Ambiente y Recursos Naturales (SEMARNAT). Jueves 30 de diciembre de 2010, Segunda Sección. 1-78 pp.

Ávila, H. 2008. Introducción al capítulo 3 Biodiversidad. In: La Biodiversidad en Aguascalientes: Estudio de Estado. Comisión para el Conocimiento y Uso de la Biodiversidad (CONABIO), Instituto del Medio Ambiente del Estado de Aguascalientes (IMAE), Universidad Autónoma de Aguascalientes (UAA). Aguascalientes, México. p. 81.

Ballesteros-Barrera, C., E. Martínez-Meyer y H. Gadsden. 2007. Effects of landcover transformation and climate change on the distribution of two microendemic lizards, genus Uma, of northern Mexico. J. Herpetol. 41: 733-740. 
Bravo Hollis, H. 1978. Las cactáceas de México. Vol. I. Universidad Nacional Autónoma de México. México, D.F. México. 746 pp.

Comisión Nacional para el Conocimiento y Uso de la Biodiversidad (CONABIO). 1999. "Uso de suelo y vegetación modificado por CONABIO". Escala 1: 1000000. Comisión Nacional para el Conocimiento y Uso de la Biodiversidad. Ciudad de México, México.

Del Castillo, R. F. y S. Trujillo. 1991. Ethnobotany of Ferocactus histrix and Echinocactus platyacanthus (Cactaceae) in the Semiarid Central Mexico: Past, present and future. Econ. Bot. 45(4): 495-502.

De la Cerda, M. 1999. Cactáceas de Aguascalientes. 2da. edición. Universidad Autónoma de Aguascalientes. Aguascalientes, México. 98 pp.

Fitz Maurice, W. A. 1989. Field notes. New locations for Mammillaria perezdelarosae. Cact. Succ. J. 61(6): 265-267.

Godínez-Álvarez, H., T. Valverde y P. Ortega-Baes. 2003. Demographic trends in the cactaceae. Bot. Rev. 69: 173-203.

Graham, C. H., S. Ferrier, F. Huettman, C. Moritz y A. T. Peterson. 2004. New developments in museum-based informatics and applications in biodiversity analysis. Trends Ecol. Evol. 19: 497-503.

Guisan, A., O. Broennimann, R. Engler, M. Vust, N. G. Yoccoz, A. Lehmann y N. E. Zimmermann. 2006. Using niche-based models to improve the sampling of rare species. Conserv. Biol. 20: 501-511.

Guisan, A. y N. E. Zimmermann. 2000. Predictive habitat distribution models in ecology. Ecol. Model. 135: 147-186.

Hernández, H. M. y H. Godínez. 1994. Contribución al conocimiento de las cactáceas amenazadas. Acta Bot. Mex. 26: 33-52.

Hijmans, R. J., S. E. Cameron, J. L. Parra, P. G. Jones y A. Jarvis. 2005. Very high resolution interpolated climate surfaces for global land areas. Int. J. Climatol. 25: 1965-1978.

Kozak, K. H., C. H. Graham y J. J. Wiens. 2008. Integrating GIS-based environmental data into evolutionary biology. Trends Ecol. Evol. 23: 141-148.

Liu, C., P. M. Berry, T. P. Dawson y R. G. Pearson. 2005. Selecting thresholds of occurrence in the prediction of species distributions. Ecography 28: 385-393.

Martin, R. 2013. Cactus and Succulent Field Number Finder. Cardiff University. Wales, UK. http://ralph.cs.cf.ac.uk/Cacti/finder.html; consultado el 14 de enero 2013.

Pearson, R. G., C. J. Raxworthy, M. Nakamura y A. Townsend Peterson. 2007. Predicting species distributions from small numbers of occurrence records: a test case using cryptic geckos in Madagascar. J. Biogeogr. 34: 102-117.

Pearson, R. G., W. Thuiller, M. B. Araujo, E. Martínez-Meyer, L. Brotons, C. McClean, L. Miles, P. Segurado, T. P. Dawson y D. C. Lees. 2006. Model-based uncertainty in species range prediction. J. Biogeogr. 33: 1704-1711.

Peterson, A. T. 2003. Predicting the geography of species' invasions via ecological niche modeling. Q. Rev. Biol. 78: 419-433.

Peterson, A. T. y D. A. Kluza. 2003. New distributional modelling approaches for GAP analysis. Anim. Conserv. 6: 47-54. 
Peterson, A. T., C. Martínez-Campos, Y. Nakazawa y E. Martínez-Meyer. 2005. Timespecific ecological niche modeling predicts spatial dynamics of vector insects and human dengue cases. Trans. R. Soc. Trop. Med. Hyg. 99: 647-655.

Peterson, A. T., M. A. Ortega-Huerta, J. Bartley, V. Sánchez-Cordero, J. Soberón, R. H. Buddemeier y D. R. B. Stockwell. 2002. Future projections for Mexican faunas under global climate change scenarios. Nature 416: 626-629.

Peterson, A. T., J. Soberón y V. Sánchez-Cordero. 1999. Conservatism of ecological niches in evolutionary time. Science 285: 1265-1267.

Phillips, S. J., R. P. Anderson y R. E. Schapire. 2006. Maximum entropy modeling of species geographic distributions. Ecol. Model. 190: 231-259.

Phillips, S. J. y M. Dudík. 2008. Modeling of species distributions with Maxent: new extensions and a comprehensive evaluation. Ecography 31: 161-175.

Phillips, S. J., M. Dudik y R. E. Schapire. 2004. A maximum entropy approach to species distribution modeling. Proceedings of the Twenty-First International Conference on Machine Learning. Association for Computing Machinery. New York. USA. p. 83.

Pilbeam, J. 1999. The Cactus file handbook Mammillaria. Nuffield Press. Oxford, UK. 380 pp.

Pilbeam, J. y D. Bowdery. 2005. Ferocactus. British Cactus and Succulent Society. 116 pp.

Quezada, E. 2008. Estudio de caso: la familia Cactaceae en el estado de Aguascalientes. In: La Biodiversidad en Aguascalientes: Estudio de Estado. Comisión Nacional para el Conocimiento y Uso de la Biodiversidad (CONABIO), Instituto del Medio Ambiente del Estado de Aguascalientes (IMAE), Universidad Autónoma de Aguascalientes (UAA). Aguascalientes, México. 117 pp.

Quezada, E., J. García Santibáñez y R. Díaz López. 2000. Nomenclator: Cactáceas en Aguascalientes. Instituto Nacional de Investigaciones Forestales, Agrícolas y Pecuarias. México, D.F., México. 47 pp.

Sánchez-Cordero, V. y E. Martínez-Meyer. 2000. Museum specimen data predict crop damage by tropical rodents. Proc. Natl. Acad. Sci. USA 97: 7074-7077.

Valiente-Banuet, A., A. Bolongaro-Crevenna, O. Briones, E. Ezcurra, M. Rosas, H. Nunez, G. Barnard y E. Vázquez. 1991. Spatial relationships between cacti and nurse shrubs in a semi-arid environment in central México. J. Veg. Sci. 2: 15-20.

Wiens, J. J. 2004. Speciation and ecology revisited: Phylogenetic niche conservatism and the origin of species. Evolution 58(1): 193-197. 